Fecha de recepción: febrero 2017 Fecha de aceptación: agosto 2017 Versión final: septiembre 2018

\section{Prospectiva latinoamericana desde la filosofía del Diseño Social}

Resumen: El artículo plantea la importancia de asumir un rol activo, en América Latina, en la edificación de un futuro colectivo. En este sentido se analiza la vinculación estrecha entre la metodología de la Prospectiva y el Diseño Social, capaz de producir innovación social a partir de la aplicación del ejercicio proyectual centrado en las necesidades locales. Asimismo se examina la importancia de superar la etapa de la innovación para alcanzar el impacto social; o dicho en otros términos, lograr que la innovación se colectivice produciendo mejoras en la calidad de la vida de las personas y propiciando el desarrollo local.

Palabras clave: Diseño social - Prospectiva - Innovación social - Impacto social - Desarrollo local.

[Resúmenes en inglés y portugués en las páginas 37-38]

${ }^{(*)}$ Doctoranda en Ciencias Políticas y Relaciones Internacionales (Universidad Complutense de Madrid, España). Master en Relaciones Internacionales (Università di Bologna, Italia, 2007). Licenciada en Relaciones Públicas (UP, 2005). Forma parte del Cuerpo Académico de la Maestría en Gestión del Diseño. Coordina el Programa de Investigación y Desarrollo en Diseño Latino y dirige la Línea de Investigación Presente y Futuro del Diseño Latino.

\title{
Introducción
}

Aún a riesgo de resultar conceptualmente tautológico, existe cierta convención en llamar Diseño Social a aquel que se focaliza en resolución de los problemas sociales y, prioritariamente, aquellos surgidos en su contexto inmediato. La denominación intenta demarcar -a veces más eficientemente que otras- la diferencia con aquellas otras aplicaciones disciplinares que se abocan al desarrollo de productos y servicios comerciales.

El diseño social consigue su fin a través de la innovación social, es decir por medio de la generación de respuestas inéditas a necesidades colectivas que mejorar sustancialmente la vida de las personas, de una comunidad, de un barrio, de una ciudad, de una región.

La amplitud y el alcance que la innovación en diseño produce, sin embargo, no es un dato menor. Sólo puede hablarse de innovación social, o de innovación en términos sociales, cuando la mejora generada sobrepasa el núcleo primario para la que originalmente fue 
proyectada y cuando sus beneficios se extienden para el resto de la comunidad, produciendo impacto social.

Dicho de otro modo, no toda innovación se colectiviza produciendo cambios en la calidad de la vida de las personas, generando impacto social y por ende, desarrollo local.

Esta situación tiene como marco y contexto la globalización y sus múltiples dialécticas contradictorias, tanto en lo cultural como en lo social y lo económico, que tensan las relaciones y la convivencia entre lo universal y lo regional.

En este sentido y en el contexto de la prospectiva, el desarrollo local y el diseño social en América Latina se posicionan como un par conceptual casi indisociable, que genera no solo nuevas miradas sobre el quehacer disciplinar y sino también sobre cómo dar respuesta a las necesidades de la región.

\section{La diversidad de la identidad común}

El ejercicio de imaginación de una prospectiva disciplinar propia en el continente implica, directa e inevitablemente, preguntarse si existe un punto de partida común, y aún más si existe un Diseño Latino o Latinoamericano.

La fórmula nominativa utilizada, muchas veces, por comunidad conceptual y para generar una suerte denominación de origen suele condensar un sinnúmero de realidades no siempre equiparables. En este sentido, encontrar una homogeneidad proyectual y un desarrollo disciplinar parejo en todos los países de la región es imposible, y hablar de una estética del diseño latinoamericano "además de equívoco resultaría pretencioso". (Álvarez, 2011, p. 49) Sin embargo, la disciplina posee "una realidad con un grado de complejidad que va mucho más allá de un mero hecho de estilo" (Bonsiepe, 2008, p. 10). Los presentes del diseño son diversos pero "existen ciertas similitudes y afinidades estructurales (que) la mirada local, a veces, desconoce”. (Fernández, 2008, p. 18)

En este sentido, el intento de generar un paneo de la realidad regional, aún en el reconocimiento de la heterogeneidad, resulta útil. En la realización de este ejercicio paranorámico, la nominación de Diseño Latino es eminentemente aspiracional y conlleva e implica la búsqueda de una forma propia de desarrollo conceptual y epistemológico en base a las realidades, problemas, necesidades e historia latinoamericana.

\section{Soberanía proyectual latinoamericana: entre el diseño contextual y el diseño social}

El desarrollo y la consolidación del diseño en América Latina, en los últimos años, han dado lugar una nueva perspectiva disciplinar que no solamente surge como respuesta frente a las demandas locales sino también que emerge como resultante de una disciplina que por primera vez puede pensarse, concebirse y producir a partir de su propia idiosincrasia identitaria.

La realidad latinoamericana contemporánea, sin embargo, pone de manifiesto la rigidez existente entre lo global y lo local, dos instancias espaciales y geográficas -a priori- com- 
plementarias, pero muchas veces interpretadas en términos de variables binarias mutuamente excluyentes. De esta forma, tal como sostiene Boiser, las opiniones quedan divididas "entre los anti-globalización y los pro-globalización. (...) Los primeros quieren tapar el sol con una mano y los segundos quieren imponer un marco ideológico y político". (Boisier, 2005, p. 48)

Esta disquisición que ataña sobretodo al campo de lo político, lo económico, lo social y lo cultural, tampoco escapa al campo del diseño, que empieza a plantearse si debe formar profesionales preparados para responder a las demandas de productos y servicios globales o a las locales y regionales con sus requerimientos específicos. Comienzan a surgir voces que sostienen que "más que la 'originalidad por la originalidad' (...) serán las soluciones originales adecuadas al contexto particular de su aplicación las que se verán favorecida”. (Baur, 2008, p. 236)

Estas nuevas posiciones intentan revalorizar y rescatar la realidad productiva y cultural local e implementar estrategias de desarrollo glocal. Es decir, propiciar un circulo virtuoso en el que "la identidad se construye en un intercambio permanente de ideas que salieron de otros contextos" (Bonsiepe, 2008, p. 13). Lo interesante de estas posturas intermedias es que entienden que "la particularidad no niega en absoluto la existencia de una cantidad de elementos sujetos al mercado global". Es la noción de contexto la que "permite no oponer de manera simplista lo global a lo local. Por eso no niega la importancia del lugar de uso como parámetro de contextualización" del diseño. (Baur, 2008, p. 236)

\section{Diseño de transformación: de la innovación al impacto social}

Para comprender la relevancia contextual actual y potencial de la innovación social vinculada al diseño (o de la innovación vinculada al diseño social), es necesario advertir brevemente sobre la evolución histórica que sufrió el término. Su interrelación con los aspectos sociales es relativamente contemporánea y un nuevo descubrimiento en su constitución terminológica.

Si durante buena parte del Siglo XX el concepto estuvo profundamente vinculado a la industria y su auge, y en la última década del siglo pasado relacionado a los aspectos tecnológicos, es recién en la actualidad que surge una imbrincancia directa con el diseño y sobre todo con la idea de transformación social (Álvarez, 2011). En relación a esta tríada, Baur sostiene que "el diseño transforma una situación por medio de la creación", y agrega que "no existe diseño sin transformación, o mejor aún, sin voluntad de transformación" (Baur, 2008, p. 233), podría decirse sin intensión de innovación.

El concepto de innovación social "designa nuevas formas organizacionales y prácticas sociales que contribuyen al mejoramiento de una situación o dan respuesta inédita a una necesidad colectiva”. (Richer, 2005, p. 51)

El paso de la simple innovación a la innovación social es el que va desde la forma absolutamente diferente y optimizada de realizar un procedimiento en un sector reducido, a la mejora que sobrepasa el núcleo primario para la que fue originalmente proyectada alcanzando al resto de la comunidad. Según la CEPAL, para que una innovación sea considerada social debe beneficiar a un grupo significativo de personas, surgir de los propios 
interesados y sus entidades de apoyo, ser original y no tratarse de experiencias repetidas, haber superado la fase de experimentación y estar consolidada, ser extrapolable y, sobre todo, ser una experiencia transformadora generando desarrollo local.

En general, el diseño no logra generar innovación social si no tiene plena conciencia de que el proceso no termina en el momento proyectual. No basta diseñar atendiendo a las necesidades locales para generar un mejoramiento de la calidad de vida, sino comprender que los mecanismos de innovación social están estrechamente vinculados al desarrollo iterativo. Es decir, requieren un ida y vuelta con el entorno que valida o modifica el rumbo del proyecto. En este sentido, puede afirmarse que no se trata de un proceso "unidireccional, sino cíclico, con numerosas causas y efectos, con progresos y retrocesos, e incluye la participación de agentes externos que han complementado o reanimado los conocimientos anterior y local, puestos en juego por todo proceso innovador". (Rodríguez Herrera y Alvarado Ugarte, 2008, p. 34)

El usuario no solo debe participar en el momento de testear el producto final, sino que debe además ser parte de su desarrollo proyectual. La participación activa garantiza no sólo que el diseño resultante sea lo más ajustado posible a las necesidades del beneficiario sino también que la mejora resulte sostenible, que el usuario la adopte como propia y que sea capaz de asumir el rol de sujeto de la innovación.

La innovación social extendida produce impacto social, y por ende desarrollo local, es decir que impulsa un proceso "capaz de promover el dinamismo económico y la mejoría de la calidad de vida de la población” (Buarque, 1999, p. 9). Sólo puede producir transformación social cuando se registra multiplicación de la experiencia, expansión de los beneficios, y sustentabilidad del proceso. Y como bien señalan Rodríguez Herrera y Alvarado Ugarte "aunque el éxito de una innovación particular no dependa de su réplica, esta le proporciona un sentido social". (2008, p. 22)

El desarrollo local puede ser visto desde tres perspectivas. Por un lado como matriz de estructuras productivas (es decir, en lo referido a los clusters industriales, según la terminología de Porter); por otro como proceso endógeno de cambio estructural (vinculado a la capacidad local para tomar decisiones, frente a opciones de desarrollo y con una mirada en la descentralización del proceso); y por último como un proceso de empoderamiento de la sociedad local (que intenta resolver, o al menos manejar, la tensión interdependiente entre devolución y globalización). (Boisier, 2005, pp. 52-55)

En el marco de este artículo interesan, particularmente, las dos últimas perspectivas, entiendo que por un lado es importante la revalorización del diseño contextual y el diseño social, y por otro que "para competir exitosamente en una economía globalizada, los territorios necesitan cada vez más políticas que ayuden a construir y explotar las capacidades endógenas". (Boisier, 2005, p. 55)

\section{El diseño social en la construcción de escenarios}

Si hay un diseño que puede pensarse como herramienta de construcción de escenarios futuros es el diseño social. 
Esto no quiere decir que el Diseño Comercial, sólo por usar la reducción terminológica simplista y contrapuesta, no pueda producir cambios. De hecho, los genera constantemente al enfrentarse a las demandas de un mercado globalizado y altamente competitivo, produciendo innovaciones industriales, morfológicas, funcionales. En este tipo de diseño el centro de la innovación es el producto o el servicio diseñado, y el destinatario es el consumidor.

El Diseño social, en cambio, pretende resolver -como fin último- problemas de una comunidad en su conjunto. Busca no solo la innovación social sobre un problema determinado en el presente sino la extrapolación gradual de las experiencias hacia el resto del colectivo y hacia el futuro, mejorando globalmente la calidad de vida de los ciudadanos o, en otras palabras, produciendo impacto social. El centro de la innovación, en este caso, es la realidad social y el destinatario último es la sociedad.

Por este motivo, la prospectiva (en el sentido metodológico) encuentra en el diseño social, y en la aplicación de la metodología del diseño a la resolución de problemáticas sociales, un aliando para el desarrollo de futuros deseables.

La prospectiva, lejos de ser una metodología de análisis pasivo, se posiciona como una forma de generar cambios sociales. No intenta saber qué sucederá, sino identificar qué se quiere que suceda para poder accionar en el presente. Es decir, sobrepasa ampliamente el campo de acción del análisis de tendencias para diseñar acciones que produzcan impacto social.

La técnica de construcción de escenarios futuros de la prospectiva es, por antonomasia, una forma de diseño social, que "usa los recursos de la utopía en cuanto 'remontar el vuelo imaginativo', pero en el proceso creativo 'articula esfuerzos concretos para transformar la realidad". (Ministerio de Desarrollo Social de Chile, 2005, p. 10)

Esta metodología tiene como base la concepción de que el futuro aún no existe y que puede crearse en base a las decisiones tomadas en el presente. Tal como sostiene Ezio Manzini es necesario hacer las cosas de una manera (radicalmente) diferente de lo que lo haría el promedio para lograr, verdaderamente, producir cambios. Es decir, para generar discontinuidades locales, "algo que quiebre la rutina proponiendo formas de comportamiento radicalmente nuevas”. (Manzini, 2015, p. 13)

Es en este contexto que el diseño social pasa a ser una herramienta útil y central para reinterpretar y resignificar soluciones ya existentes, y para reposicionar a los actores sociales de un rol marginal a un papel activo en la construcción de su propio futuro.

De esta forma, cuando se persigue la innovación y el consecuente impacto social, ya no sólo pensando en una necesidad presente sino dirigiendo los esfuerzos hacia el futuro que se quiere alcanzar, la prospectiva y el diseño se enlazan, retroalimentan y potencian generando sinergia social.

\section{Panorámica de futuros comunes y responsabilidad de las universidades}

La panorámica de la prospectiva latinoamericana no busca homogenizar el porvenir, sino acodar ciertos lineamientos comunes hacia donde conducir los esfuerzos.

Si bien la construcción de este futuro está influenciada por un sinnúmero de factores, muchas veces exógenos y poco controlables, la formación de diseñadores capaces de atender 
a las necesidades sociales actuales de la región y concientes de su rol activo en la modificación de la realidad debería ser parte del aspiracional de las instituciones de educación superior, para que estos puedan ser "sujeto y no objeto de la globalización". (Boisier, 2005, p. 57) Resulta asidua la discusión sobre la necesidad de que la pedagogía del diseño se centre en el desarrollo de competencias profesionales para que los egresados puedan enfrentarse a los nuevos desafíos que el mercado impone. Sin embargo, son menos frecuentes las reflexiones entorno a la importancia de formar diseñadores que puedan pensarse no solo como individuos aislados frente a los desafíos laborales, sino también como miembros activos de una sociedad a la que pueden contribuir desde su perspectiva profesional.

En este sentido, no puede negarse el grado de responsabilidad de las universidades en la configuración del futuro y por ende, en la reflexión sobre el presente curricular conciente de su pertenencia regional y de las necesidades continentales. Es decir, de la pertenencia entendida "como la incrustación en la comunidad local". (Boisier, 2005, p. 59)

Sin embargo, no se puede esperar de la universidad -como institución- todo el mecanismo de generación del conocimiento y la formación para el cambio social. En lo que respecta a la prospectiva y al diseño social aplicado, las usinas de pensamiento, las redes cognitivas, los centros de innovación, son también núcleos de generación de innovación y pueden ser también parte de la realidad de un continente comprometido con su propia realidad, y con sus escollos en cuanto al desarrollo sostenido.

\section{Conclusiones}

Puede sostenerse que el ejercicio prospectivo, en combinación con el diseño social, permite adoptar un rol activo en el desarrollo de la realidad continental.

Si bien, esta afirmación puede plantearse sin mayores reservas, no así la implementación de la metodología. El primer escollo surge del requerimiento de identificar un punto de partida compartido para poder efectuar acciones tendientes a alcanzar el futuro deseado. Parece menos problemático el aspiracional de un futuro latinoamericano común que la compleja definición de las diversas realidades contemporáneas del diseño en cada uno de los países de la región.

Se puede argumentar, como primer intento para retirar de la discusión este planteamiento aparentemente irresoluble, que el presente no es común por ser homogéneo, sino por ser regional o geográfico. Y que la diversidad latinoamericana, paradójicamente, es parte de la identidad compartida, sin que por ello quede invalidada.

En el mismo sentido, la nominación "Diseño Latino" o "Diseño Latinoamericano" no adjetiva la uniformidad, analogía o similitud, sino más bien los repetidos intentos de desarrollo de una epistemología disciplinar propia. Es una interpretación determinada por el continente y no por el contenido.

Más aún, la prospectiva latinoamericana, conciente de sus múltiples realidades de origen, tendrá que desplegar un sinnúmero de acciones diferentes (atendiendo a esos presentes diversos) si quiere alcanzar un futuro común. En ese ejercicio de intervención, el diseño contextual y, sobre todo, el diseño social dan a Latinoamérica una importante soberanía proyectual, sin que este mirar introspectivo hacia las necesidades y requerimientos locales 
signifique olvidar el contexto amplio y globalizado (que influencia, condiciona y, a veces, colabora).

Por último, puede que concluirse que pensar y concebir el diseño social desde la prospectiva y no desde las tendencias, es pasar de la pasividad de los hechos predeterminados a sucederse a la proactividad de la construcción y consolidación de un futuro regional, donde el diseño de innovación social contribuya al desarrollo latinoamericano.

\section{Bibliografía}

Álvarez, P. (2011). “Diseño Latinoamericano. Presente y Pasado”, en Revista Diseña (número 3, pp. 44-49).

Baur, R. (2008). “Diseño global y diseño contextual”, en Bonsiepe, G. y Fernandez, S. (2008). Historia del Diseño en Ámerica Latina y el Caribe. Industrialización y comunicación visual para la Autonomía. Sao Paulo: Blücher.

Boiser, S. (2005). “¿Hay espacio para el desarrollo local en la globalización?”, en Revista de la CEPAL (número 86, agosto de 2005, p. 47-62).

Bonsiepe, G. (2008). "Prefacio", en Bonsiepe, G. y Fernandez, S. (2008). Historia del Diseño en Ámerica Latina y el Caribe. Industrialización y comunicación visual para la Autonomía. Sao Paulo: Blücher.

Bonsiepe, G. y Fernandez, S. (2008). Historia del Diseño en Ámerica Latina y el Caribe. Industrialización y comunicación visual para la Autonomía. Sao Paulo: Blücher.

Buarque, S. (1999). Metodologia de planejamento do desenvolvimento local e municipal sustentável. Brasília: INCRA/IICA.

Fernandez, S. (2008). "Presentación”, en Bonsiepe, G. y Fernandez, S. (2008). Historia del Diseño en Ámerica Latina y el Caribe. Industrialización y comunicación visual para la Autonomía. Sao Paulo: Blücher.

Manzini, E. (2015). Design, When Everybody Designs: An Introduction to Design for Social Innovation. Massachusetts: MIT Press.

Ministerio de Desarrollo Social de Chile (2005). Prospectiva y construcción de escenarios para el desarrollo territorial. Santiago: Gráfica Fanny.

Richer, M. (2005). "Innovación social y desarrollo local en un municipio andino", en Revista Venezolana de Economía Social (año 5, número 9, pp. 49-65).

\footnotetext{
Abstract: The paper discusses the importance of assuming an active role in Latin America's collective future construction. In this sense, it is analyzed the close link between the methodology of Foresight and Social Design, capable of producing social innovation from the application of the design exercise focused on local needs.

It also examines the importance of overcoming the stage of innovation to achieve social impact, or in other words, to ensure that innovation is collectivized by producing improvements in the quality of people's lives and by fostering local development.
} 
Key words: Social design - Foresight - Social innovation - Social impact - Local development.

Resumo: $\mathrm{O}$ artigo discute a importância de se ter um papel ativo na construção de um futuro coletivo na América Latina. Neste sentido, analisa a estreita ligação entre a metodologia Prospectiva e o Design Social, capaz de produzir inovação social a partir da aplicação do exercício projetual focado nas necessidades locais.

$\mathrm{O}$ artigo ainda no artigo discute a importância de superar o estágio de inovação para alcançar impacto social; ou em outras palavras, coletivizar a inovação, produzindo melhorias na qualidade de vida das pessoas e promovindo o desenvolvimento local.

Palavras chave: Design social - Prospectiva - Inovação social - Impacto social - Desenvolvimento local. 